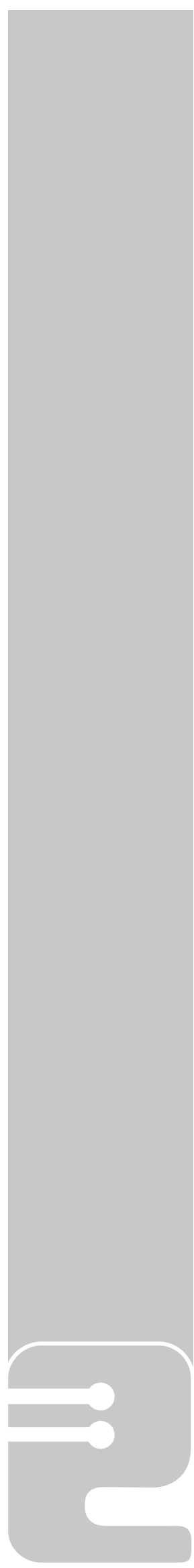

\title{
Comparación de técnicas de modelamiento para el control de procesos: un enfoque de aprendizaje con dinámica de sistemas
}

\author{
Jairo Vargas Caleño ${ }^{1}$, Milton M. Herrera R²
}

Recibido: 23 de febrero de 2015 Aprobado: 24 de mayo de 2015

\section{Resumen}

El modelamiento por medio de la dinámica de sistemas para procesos físicos se puede utilizar para generar estrategias de control. Se presenta una aplicación con Simulink y Vensim para analizar el comportamiento de un sistema y generar estrategias para mantener un punto deseado. Este artículo muestra cómo utilizar la definición obtenida desde la dinámica de sistemas para modelar procesos físicos, sin un alto grado de la matemática formal y como apoyo a los procesos de aprendizaje. Se plantea un contraste entre la aplicación de técnicas de control y la simulación con dinámica de sistemas, y una discusión sobre la contribución a los procesos de aprendizaje en el modelamiento de sistemas de control.

Palabras claves: educación, control de procesos, dinámica de sistemas, controlador proporcional, controlador difuso.

\section{INTRODUCCIÓN}

Un proceso o sistema dinámico puede definirse como una combinación de elementos o componentes relacionados entre sí, que actúan para alcanzar una determinada meta. El estudio de un proceso dinámico es el análisis del cambio a lo largo del tiempo de las variables de salida, a partir de las variables de entrada que afectan el comportamiento dinámico del proceso (Norgaard, 2000). Por consiguiente, la dinámica de sistemas estudia el comportamiento de un sistema y sus variables en el tiempo (Herrera \& Orjuela, 2014; Sterman, 2000; Aracil \& Gordillo, 1997; Morecroft \& Sterman, 1994).

Desde un punto de vista matemático, un proceso dinámico se suele representar mediante una relación entre las variables de entrada y salida del proceso, es decir, entre las medibles y observables. Para la mayor parte de los procesos dinámicos reales no es fácil conocer estas relaciones o representaciones matemáticas. Generalmente, la información disponible viene

1 Ingeniero de control, aspirante al grado de magíster en Ingeniería Industrial. Ingeniero de proyectos en el sector industrial y coordinador de proyectos tecnológicos con énfasis en seguridad electrónica en los sectores bancario y comercial, en campus universitarios y conjuntos residenciales. En la parte académica trabaja actualmente en el área de dinámica de sistemas aplicada a modelos de control de procesos, modelamiento de problemas sociales y herramientas pedagógicas destinadas a cursos de electrónica e ingeniería en control. jairocale@yahoo.com.

2 Ingeniero de producción y magíster en Ingeniería Industrial de la Universidad Distrital Francisco José de Caldas. Estudiante de doctorado en modelado en política y gestión pública. Docente investigador de grupo GIP de la Universidad Católica de Colombia. Bogotá D.C., Colombia. Áreas de interés: investigación de operaciones, logística, energía y dinámica de sistemas. mmherrera@ucatolica.edu.co. 
dada por un conjunto de datos observados de entrada y salida, los cuales requieren interpretación para obtener una representación matemática del proceso.

La dificultad de determinar dicha representación, también llamada modelo del proceso, se conoce como el problema de modelización e identificación de procesos dinámicos. La dinámica de sistemas es una herramienta que posibilita la creación de modelos a partir de la identificación de los elementos y las relaciones entre ellos, propios de un proceso objeto de estudio. Por ende, cada proceso o sistema posee características que lo diferencian y que caracterizan su proceso de interacción.

El artículo muestra una representación de modelos clásicos con dinámica de sistemas que mejoran la perspectiva en el proceso de aprendizaje de los sistemas de control y su aplicación. Por lo tanto, el trabajo contribuye al modelamiento dinámico por medio de la metodología de dinámica de sistemas, enfocado en la comprensión de sistemas de control y comparado con los modelos de control de procesos empleados. Esto permite una mayor comprensión y aprendizaje por medio de la simulación con dinámica de sistemas.

En los siguientes apartados se presentarán algunos conceptos referentes a los tipos de modelos disponibles para modelar procesos dinámicos de interés en el campo del control automático, así como la aplicación del pensamiento sistémico y la metodología de la dinámica de sistemas para el desarrollo de modelos de control proporcional, integral y una variante de control difuso que emplea una metodología denominada CBR para los procesos de implicación, agregación y desfusificacion.

\subsection{Modelos para el control de procesos}

Al abordar el problema del modelamiento se debe recordar que la finalidad del modelo está en que para una serie de estímulos en la entrada, éste debe ser capaz de generar una serie de respuestas a su salida. En la figura 1 se presenta el concepto de modelo en forma gráfica. Igualmente, los modelos son representaciones del mundo real en los que confluyen variables que se relacionan entre sí (variables de entrada) por medio de un proceso particular que lo caracteriza, y se obtienen una serie de respuestas o resultados (variables de salida).

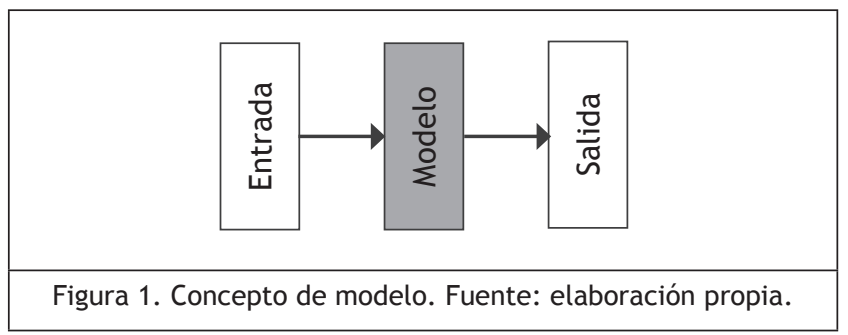

En concreto, el problema del modelamiento se ha abordado mediante diversas metodologías. De hecho, el método empleado en la construcción del modelo le otorga ciertas características, con base en las cuales es posible subdividir los tipos de modelos dentro de tres grandes grupos: modelos de caja blanca, de caja gris y de caja negra (Norgaard, 2000). Por otra parte, Sabounchi et al. (2011) proponen un modelado según la teoría difusa mediante la dinámica de sistemas con la inclusión de variables lingüísticas. Adicionalmente, Mohapatra \& Sharma (1985) desarrollaron un modelo desde la teoría de control para el diseño de políticas con un enfoque en dinámica de sistemas. El modelado de problemas de gestión de tecnología integra los sistemas de inferencia difusos y dinámica de sistemas aplicados en las cadenas de suministro (Herrera Ramírez, 2014).

Wolstenholme (1986) desarrolla un algoritmo de control con un modelo para la dinámica de sistemas. En este contexto la ingeniería de control ha optado por la aplicación y uso de herramientas computacionales como Matlab y Scilab, entre otros, para la construcción de modelos empleados en el análisis de problemas con una fuerte formalidad matemática. Sin embargo, en este artículo se propone la extensión de los modelos por medio de estas herramientas a dinámica de sistemas. En general, es común usar herramientas matemáticas como las ecuaciones diferenciales para diseñar modelos y solucionar problemas en procesos físicos. Un ejemplo de ello es el modelo matemático para un oscilador amortiguado presentado por Ogata (1998). Para la construcción del modelo del oscilador amortiguado, primero se usa la segunda ley de Newton. Combinando (1) y (2), se obtienen las ecuaciones que describen el comportamiento del sistema.

$$
\begin{gathered}
\dot{x}=v x(0)=x_{0}(1) \\
\dot{v}=-\frac{k}{m} x-\frac{B}{m} v v(0)=v_{0}(2)
\end{gathered}
$$

Empleando Simulink de Matlab se puede realizar el modelo con ayuda de las ecuaciones (1) y (2), establecidas para el oscilador amortiguado. El modelo resultante se muestra en la tabla 1 . La respuesta del modelo se obtuvo con una entrada escalón unitario.

Ahora, si se interpretan los flujos como cambios en el tiempo y la combinación de un flujo con variables asociadas como un integrador, se puede utilizar algún software de dinámica de sistemas para representar las ecuaciones que describen el comportamiento del oscilador amortiguado. En la tabla 1 se presenta un modelo para el oscilador amortiguado y su comportamiento en el tiempo, el que se asumen las constantes $\mathrm{k}$ y $\mathrm{B}$ con valores iguales a 10 y 100 , respectivamente. 


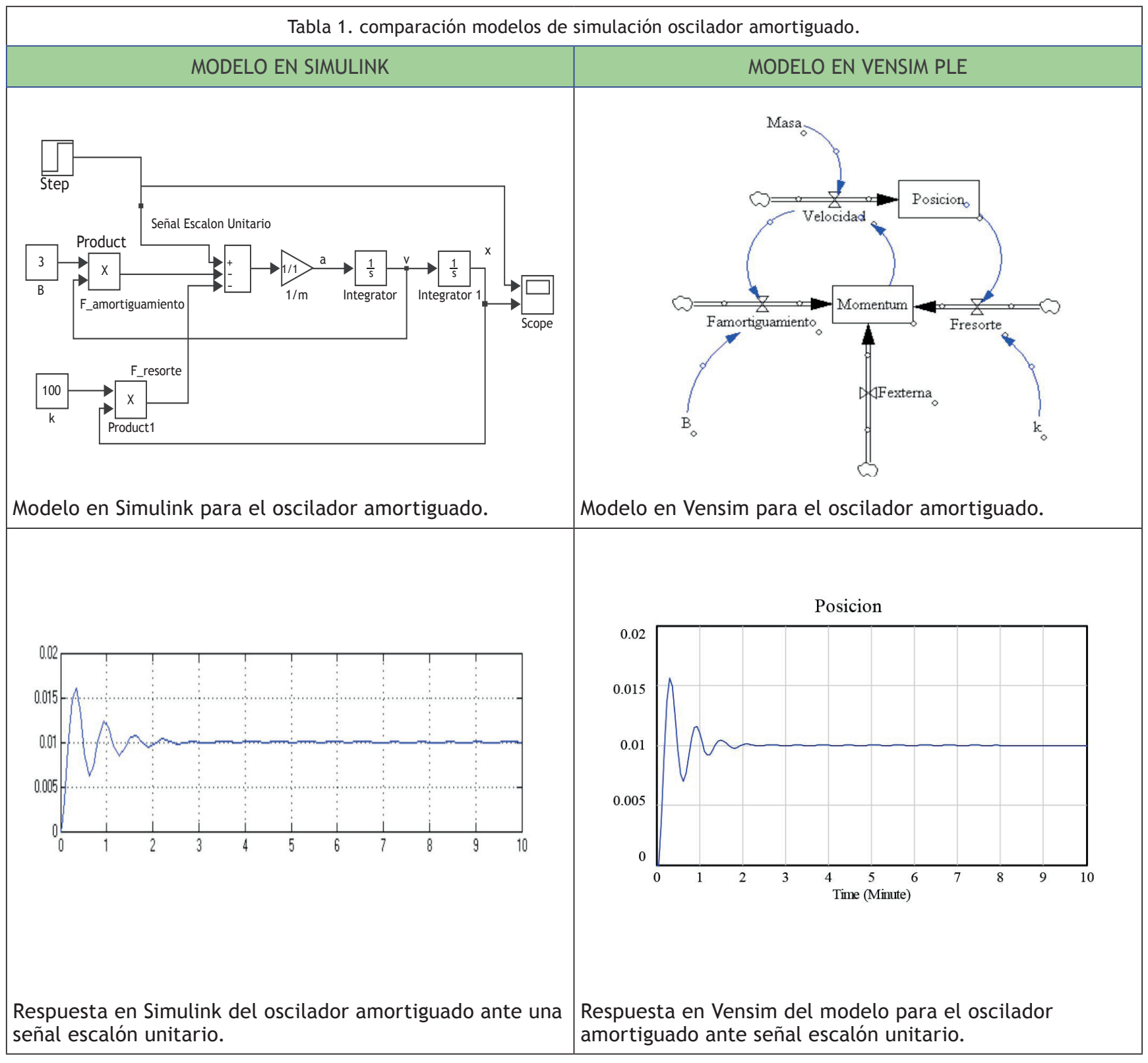

Es de notar que la respuesta de los dos modelos implementados muestran el mismo comportamiento ante la acción de una entrada escalón unitario, empleada como fuerza externa para excitar el sistema. La simulación obtenida con dinámica de sistemas resulta sencilla y con un mayor grado de flexibilidad a cambios y aumento de variables que intervienen en el sistema. Por lo tanto, la metodología con dinámica de sistemas resulta sencilla para el proceso de aprendizaje en la comprensión de un proceso físico de la mecánica clásica.

Según describe Fuchs (2006), la dinámica de sistemas se desarrolló fuera de los sistemas convencionales de la ciencia y de la ingeniería de control debido a su bajo nivel de formalismo matemático, característica propia de esta área del conocimiento y de los modelos implementados con ella. Sin embargo, la dinámica de sistemas permite también obtener modelos que

representan, con un grado de fidelidad alto, el comportamiento de sistemas, plantas o procesos comúnmente estudiados desde los enfoques de la ingeniería, principalmente la de control automático. En consecuencia, Herrera \& Echavarría (2008) presentan un modelo desde la ingeniería de control y la dinámica de sistemas para un proceso de producción hidropónico.

Por lo tanto, el modelado con dinámica de sistemas es una forma de representar un proceso físico que permite apoyar los de aprendizaje básicos en la ingeniería de control.

\subsection{Pensamiento sistémico}

Caselles (2008) describe cómo el paradigma mecanicista, fundamentado en las ideas de René Descartes, permaneció vigente durante mucho tiempo, 
permitiendo el desarrollo y la especialización del conocimiento mediante el cual se logró solucionar numerosos problemas. Sin embargo, la realidad mostró que dichos problemas se iban tornando cada vez más complejos, e hizo necesaria la participación de varios especialistas para estudiarlo, entenderlo y proponer soluciones pertinentes.

Este paradigma se caracteriza porque aborda el mundo como un gigantesco mecanismo, integrado por un conjunto de componentes que cumplen con el principio de causalidad y cuyo comportamiento se puede ajustar a leyes matemáticas. De tal forma, si se tiene la información adecuada sobre la situación de los elementos del sistema es posible determinar con precisión el comportamiento futuro de sus componentes y por ende del mismo sistema. Sin embargo, esta característica hizo que se calificara el pensamiento mecanicista como una manera de entender la realidad de forma simplificada o reducida, al considerar que el análisis de las partes es suficiente para dar cuenta de las propiedades del todo.

Si bien es cierto que las corrientes mecanicistas permitieron un desarrollo importante de la ciencia, la técnica y la tecnología, gracias al trabajo del biólogo y filosofo Ludwig von Bertalanffy, se estableció una nueva manera de concebir la realidad, lo que permitió responder incógnitas del paradigma mecanicista, y solucionar algunos problemas que éste no satisfacía de algún modo.

De esta forma, la introducción del concepto de sistema, definido por Bertalanffy como un conjunto de elementos interrelacionados, inició una nueva etapa en la que se observó el mundo de una forma sistemática y científica de aproximación y representación de la realidad y, al mismo tiempo, como una orientación hacia una práctica de formas de trabajo interdisciplinarias con una perspectiva holística, integradora, en la que priman las relaciones y los conjuntos que emergen de ellas (Caselles, 2008).

En general, el enfoque sistémico está presente en la aplicación de la teoría general de sistemas en cualquier disciplina. Abarca una amplia variedad de métodos, herramientas y principios, todos orientados a examinar la interrelación de las fuerzas que forman parte de un proceso común. En congruencia, Bajracharya (2010) muestra la relevancia del pensamiento sistémico en la toma de decisiones y la dinámica de sistemas como el método que utiliza este enfoque para solucionar problemas en procesos productivos, sociales, ambientales y económicos.

Dentro de este grupo de metodologías y técnicas se incluye la dinámica de sistemas, cuya relación con la forma de pensamiento sistémico consiste en que estudian el mismo tipo de sistemas. Sin embargo, el pensamiento sistémico suele llegar hasta la construcción de los diagramas causales del sistema, mientras que la dinámica de sistemas, avanza en la construcción y análisis de modelos que representen el sistema objeto de estudio a partir de las relaciones identificadas.

En 1969 se publicó la obra Urban Dynamics, en la que se muestra cómo los modelos llevados a cabo con dinámica de sistemas se pueden aplicar a los sistemas de las grandes ciudades del planeta. Este esfuerzo pionero culminó en 1970, cuando apareció el primer modelo del mundo (World Dynamics), trabajo que sirvió de base para que Meadows realizara el primer informe al Club de Roma, divulgado posteriormente con el nombre de "The limits to growth", en el que se concluyó que el patrón de crecimiento de la población durante el siglo $\mathrm{XX}$, el uso de los recursos no renovables, el capital y la contaminación son insostenibles de cara al siglo XXI, pues no reconoce los limites inminentes a dicho crecimiento. Este trabajo y su discusión popularizaron la dinámica de sistemas a escala mundial como un paradigma, lenguaje y metodología de estudio de sistemas complejos, no lineales con la ayuda del computador.

Es así como la dinámica de sistemas permite la comprensión de los problemas desde una óptica de sistema (conjunto de elementos que se relacionan entre sí de manera tal que un cambio en uno de ellos modifica el todo). Este enfoque permite una visión clara y realista para analizar las relaciones entre los elementos que configuran la estructura que provoca el comportamiento por estudiar, entender y en ocasiones modificar.

\subsection{Control de nivel de un fluido en un tanque basado en diagramas de Forrester}

En el trabajo que se ha desarrollado sobre la investigación en la integración de diversos métodos y su relación con la dinámica de sistemas se presenta el modelo de una planta descrito por Charles (2001), consistente en un tanque de geometría rectangular para almacenamiento de agua, el cual posee una fuente de agua y un drenaje, lo que permite distinguir un flujo de entrada y un flujo de salida sin turbulencia.

Dado que el sistema puede ser representado a partir de las variables flujo de líquido, resistencia hidráulica y capacidad de almacenamiento, la cantidad de líquido almacenado en el tanque estará dada por el flujo de entrada menos el flujo de salida, esto es:

$$
\text { C. } \frac{d h}{d t}=\mathrm{Qi}-\mathrm{Qo}
$$


C corresponde a la capacidad de almacenamiento de líquido y Qo representa el caudal de salida sin turbulencia. Remplazando, se tiene entonces:

$$
\frac{d h}{d t}=\frac{1}{c}\left(\mathrm{Qi}-\frac{h}{R_{h}}\right)
$$

La ecuación 4 representa la función de transferencia de la planta que relaciona el nivel de líquido presente en el tanque en función de los caudales de entrada y salida. Nuevamente, empleando Simulink de Matlab se implementa el modelo que representa el comportamiento de la planta en la tabla 2. Se observa que el tanque, que inicialmente se supone tiene una altura de líquido igual a $1 \mathrm{~m}$, sin presencia de caudal de entrada, se desocupa en 6 segundos, tiempo en el cual el nivel o altura de líquido es de cero $0 \mathrm{~m}$.
Ahora, teniendo en cuenta lo propuesto por Hans (2006) y empleando la dinámica de sistemas, se implementa un modelo para la misma planta, el cual consta de una variable de nivel, en función de un flujo de entrada y otro de salida. Los flujos están determinados por las variables auxiliares ki y ko, las cuales representan el porcentaje de apertura de las válvulas de entrada y salida de líquido. La variable auxiliar $\mathrm{h}(0)=1[\mathrm{~m}]$ representa la altura inicial de líquido presente en el tanque. En este caso, $\mathrm{ki}=0$ para representar la ausencia de caudal de entrada y ko=1 para indicar que la válvula de salida está totalmente abierta. En la gráfica correspondiente al nivel de líquido en el tanque se observa que alcanza una altura de líquido de cero metros, después de seis segundos.

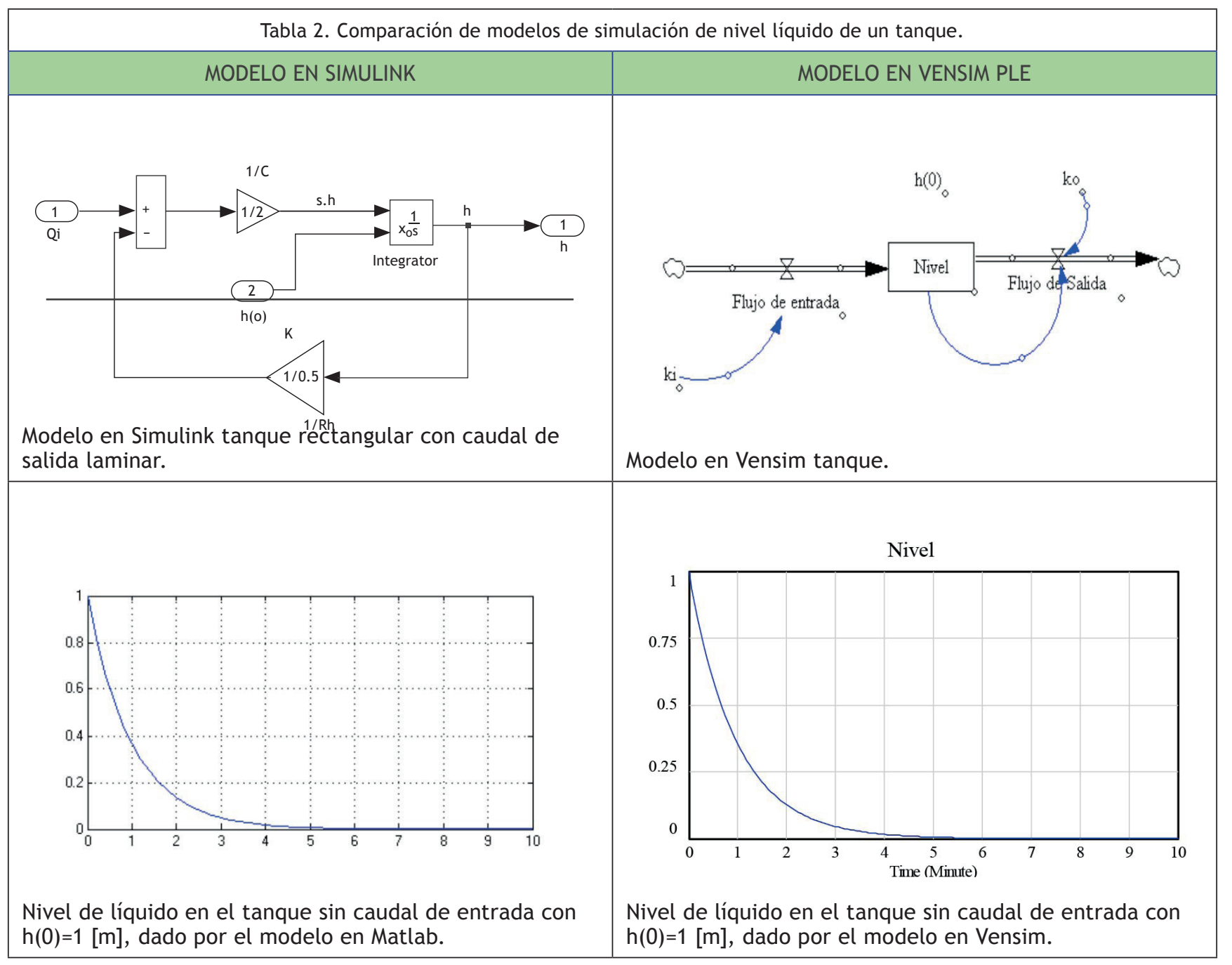

Nuevamente, se observa cómo el modelo realizado con herramientas propias de la dinámica de sistemas logra representar en una mejor medida el comportamiento de la planta; al mismo tiempo, el modelo desarrollado en Simulink con el enfoque de ingeniería de control y basado en la función de transferencia determinada de forma matemática. En resumen, se puede inferir el grado de integración y simplificación desde un modelo con un enfoque matemático y un enfoque sistémico propuesto. Por consiguiente, los procesos 
de aprendizaje empleados en la ingeniería de control pueden ser analizados desde un enfoque sistémico que permite mayor apropiación del conocimiento y las interacciones de las variables.

\subsection{Modelos para control proporcional e integral}

En el contexto del estudio de los procesos dinámicos es habitual perseguir que la salida de dicho proceso evolucione en una dirección deseada, tal como mantener una cierta condición estable o seguir una trayectoria especificada para conseguir el mejor rendimiento del proceso y una actuación adecuada. Con este propósito, los procesos dinámicos requieren lo que se conoce como sistemas de control, es decir, aquellos que mediante la manipulación de ciertas variables de entrada al proceso (las que puedan ser manipuladas), regulan el comportamiento de las variables de salida para alcanzar el objetivo deseado.

Se muestra un modelo de control (figura 2) para el ejemplo del tanque basado en Isee Systems (2012). En este modelo el objetivo consistió en alcanzar y mantener de forma estable, el nivel o altura del líquido en el tanque dado un punto de referencia u operación.

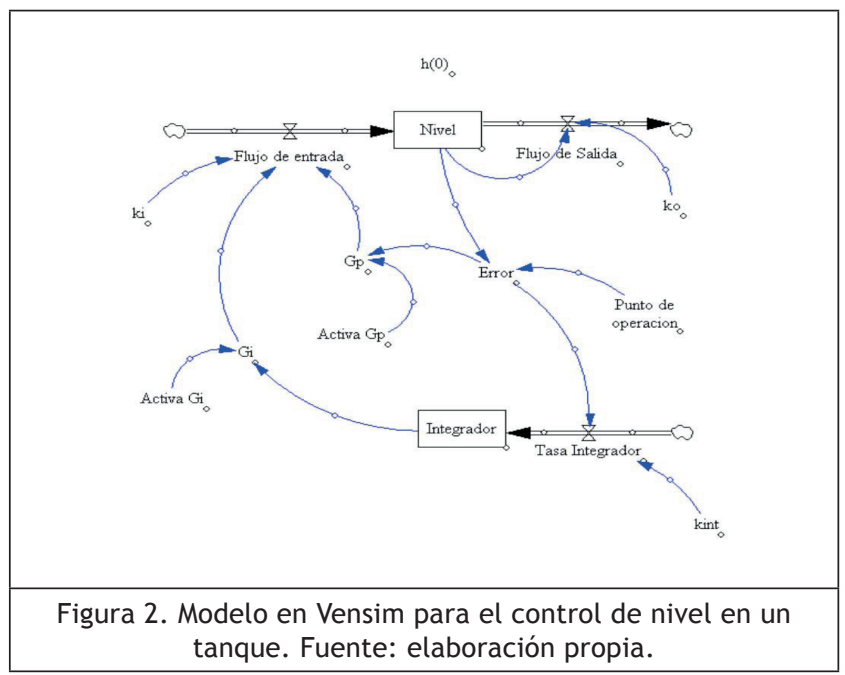

En el modelo de control se obtiene el error dado por la diferencia entre el nivel de líquido presente en el tanque y un punto de operación establecido. El error, entonces, se emplea para obtener una ganancia proporcional (Gp) y una ganancia integral (Gi). Esta última se establece por medio de una variable de flujo (tasa integrador), una constante de integración (kint) y una variable de nivel (integrador). Los valores de Gp y Gi se encargan de ajustar el flujo de entrada al tanque para mantener la altura especificada.

A continuación se presenta la respuesta de la planta, fijando un punto de operación igual a 1,5 $\mathrm{m}$ y asumiendo un nivel inicial de líquido de $1 \mathrm{~m}$, con flujos de entrada y salida iguales a la mitad de su valor máximo, es decir 0,5 (figura 3).

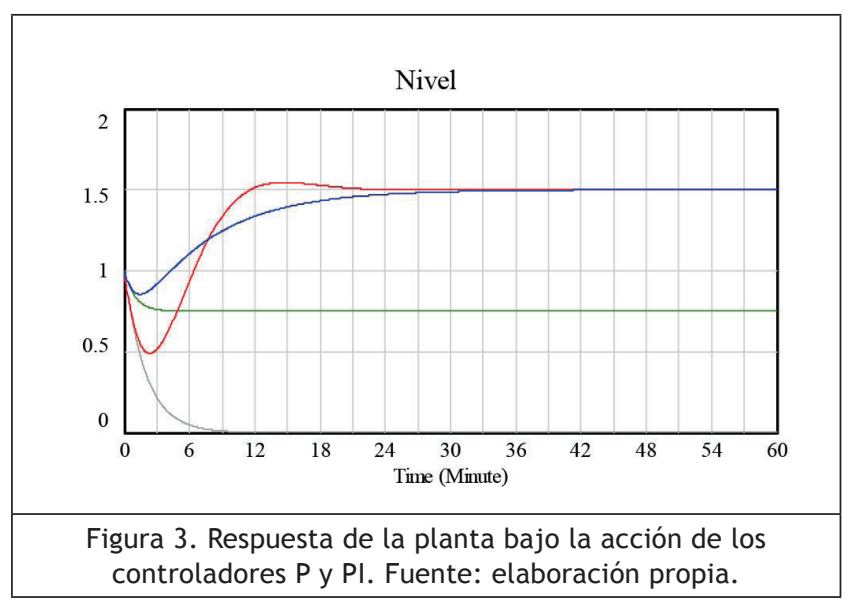

La gráfica de color gris representa la respuesta de la planta sin flujo de entrada y flujo de salida del $50 \%$. La gráfica de color verde muestra la respuesta de la planta ante la acción del control proporcional. En este caso, el nivel del tanque decae hasta un valor de 0,75 $\mathrm{m}$ y no logra estabilizar la planta en el nivel deseado. La gráfica de color rojo muestra la respuesta de la planta ante la acción del control integral. En este caso, el nivel del tanque decae hasta un valor próximo a 0,5 m hasta los 2,5 min. De ahí en adelante, el nivel empieza a subir mostrando un sobrepaso entre los minutos $12 \mathrm{y}$ 20 , para luego estabilizarse en el punto de operación.

La gráfica de color azul expone la respuesta de la planta ante la acción simultánea de los controladores proporcional e integral (PI). En este caso, el nivel del tanque decae hasta un valor próximo a $0,85 \mathrm{~m}$ hasta los 1,3 min; de ahí en adelante el nivel empieza a subir para luego estabilizarse después del minuto 33 en el punto de operación. La acción del controlador PI elimina el sobrepaso del punto de operación. Se observa el error para los cuatro casos anteriores (figura 4).

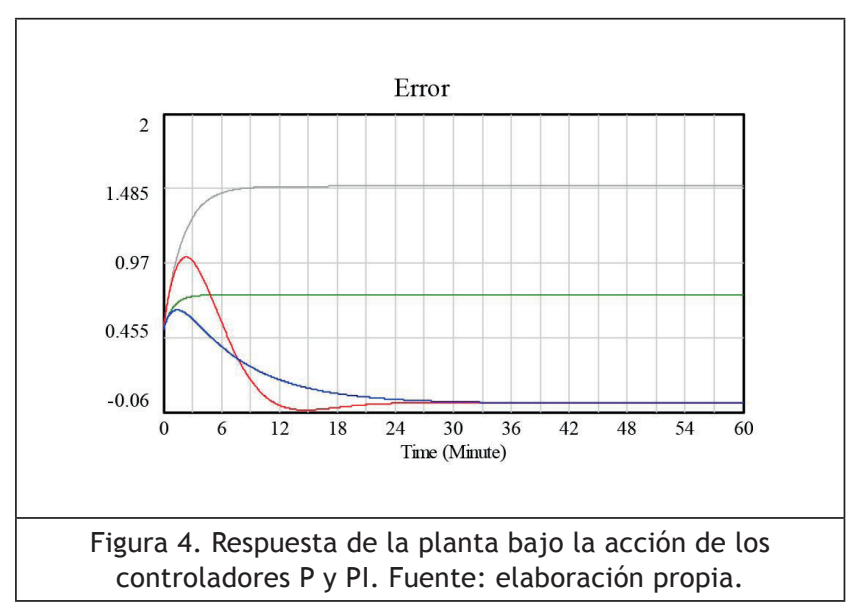


Los resultados obtenidos permiten verificar cómo las estrategias de control implementadas logran mantener la variable controlada dado un valor de referencia. Aunque no se están utilizando ecuaciones matemáticas complejas para representar la planta, se están usando las definiciones básicas de la dinámica de sistemas:

- Los niveles se acumulan o integran sus flujos.

- El flujo neto en un nivel es la tasa de cambio del nivel.

- La tasa neta de cambio de un nivel corresponde a su derivada.

En las anteriores expresiones, los niveles son generalmente una cantidad y los flujos se miden en las mismas unidades de la cantidad por unidad de tiempo. Además, según la teoría de control, los niveles únicamente cambian por flujos de entrada y salida, estos últimos determinados por los niveles.

\subsection{Modelo para control difuso}

Observada la gran versatilidad y utilidad de los controladores proporcional $(\mathrm{P})$ y proporcional-integral (PI), resulta interesante analizar un tipo de controlador que durante los últimos años se emplea con gran frecuencia debido a los buenos resultados que presenta.

Los controladores difusos corresponden a una de las aplicaciones más comunes de la lógica difusa (Del Brío, 2001). Éstos se implementan atendiendo al conocimiento de un experto, expresando dicho conocimiento con variables lingüísticas representadas por conjuntos difusos. En Wang (1997) se describen varias técnicas que ofrecen buenos resultados si se emplean de forma apropiada. En general, el controlador difuso queda determinado por las reglas de control establecidas con los antecedentes y consecuentes que representen el comportamiento deseado.

Para continuar con el ejemplo de control de nivel en el tanque desarrollado, se definen las siguientes reglas:

- R1: Si el nivel es muy bajo entonces el flujo es muy alto.

- R2: Si el nivel es bajo entonces el flujo es alto.

- R3:Si el nivel es alto entonces el flujo es bajo.

- R4: Si el nivel es muy alto entonces el flujo es muy bajo.

- Las funciones de pertenencia que representan las variables nivel y caudal se muestran en las figuras 5 y 6 , respectivamente.
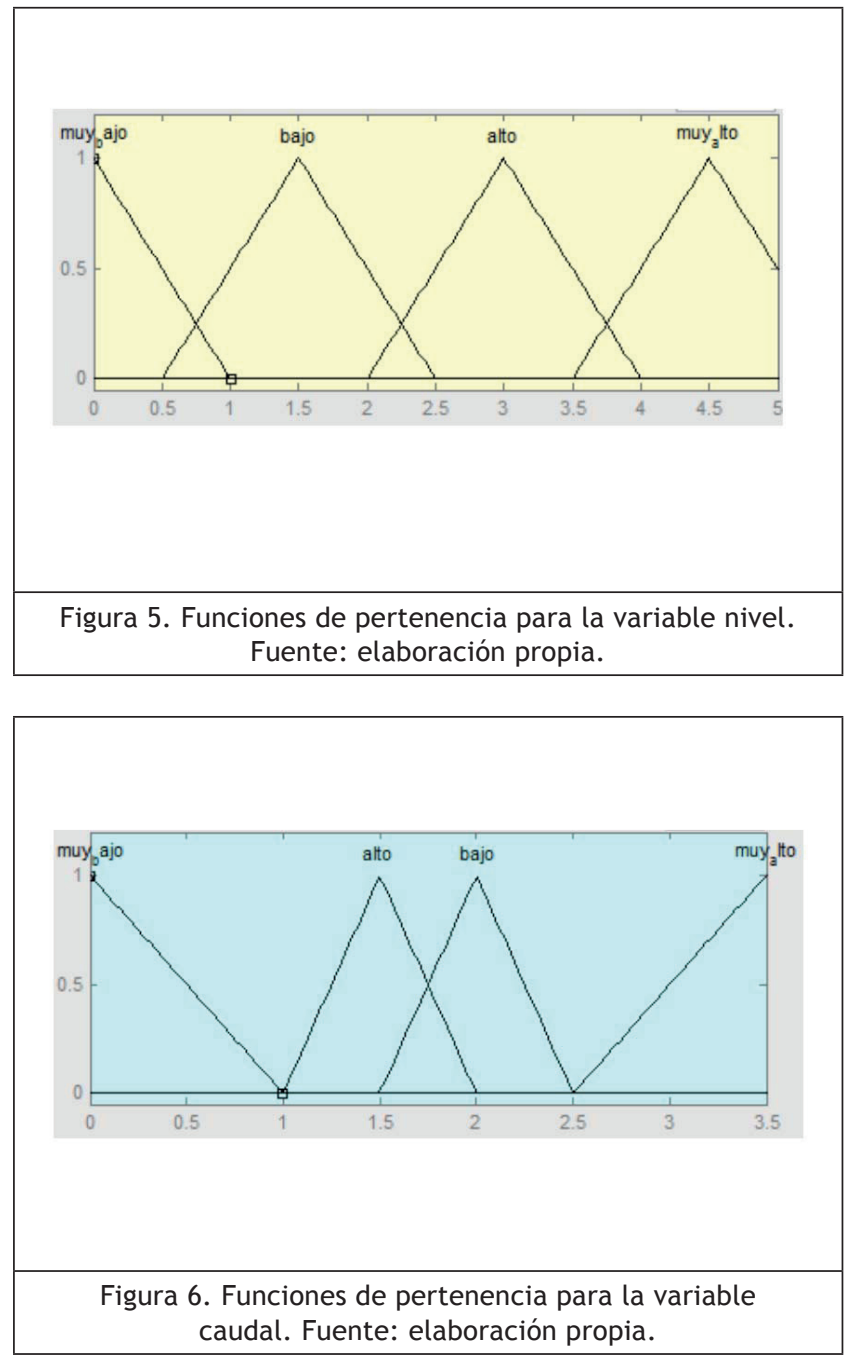

La estructura del controlador difuso implementado en Matlab, se muestra en la figura 7.

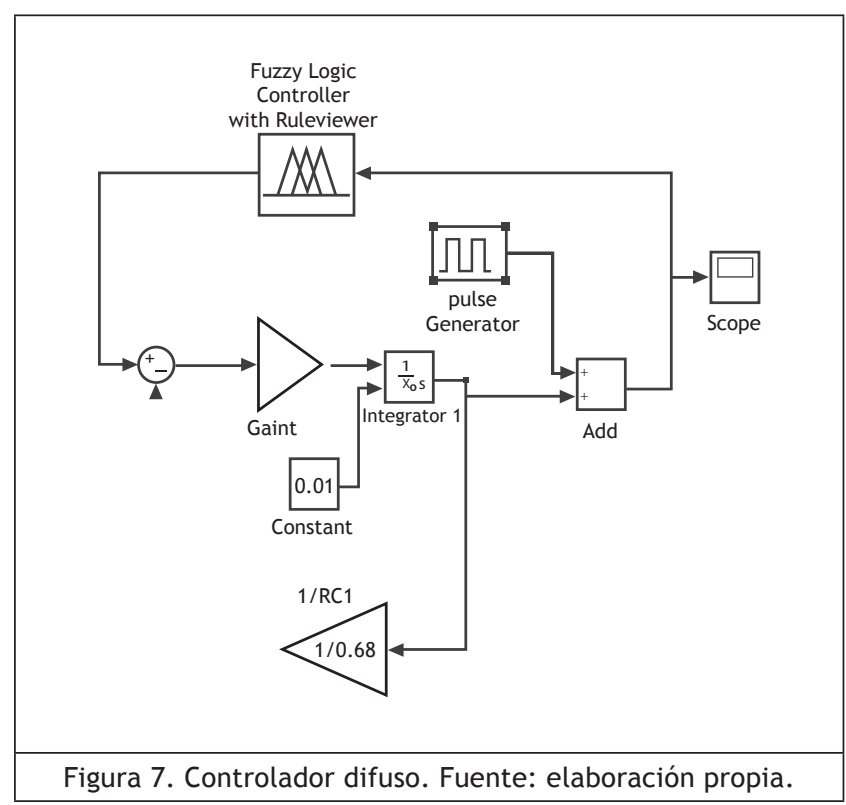


Llevando a cabo el proceso de simulación, la respuesta obtenida de la planta con el controlador difuso es la que aparece en la figura 8.

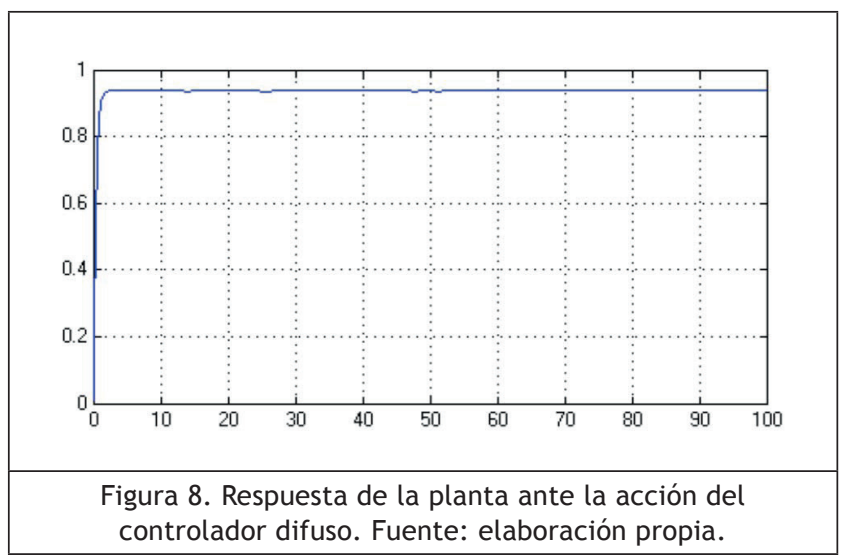

Adicionando al modelo una perturbación, empleando un tren de pulsos con una amplitud y frecuencia específicos, la respuesta de la planta cambia a la mostrada en la figura 9.

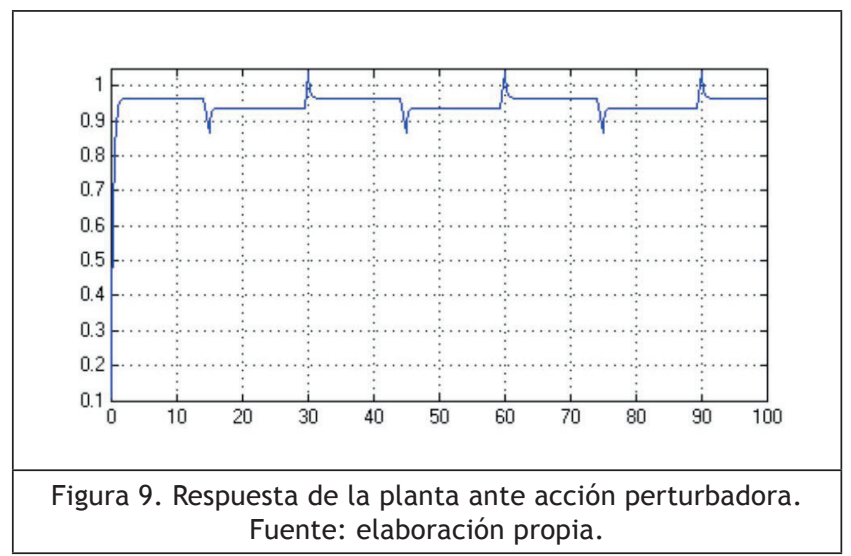

Las gráficas obtenidas muestran cómo el nivel se estabiliza en un valor cercano a $1 \mathrm{~m}$ de altura y ante la presencia de la señal perturbadora el controlador permite mantener el nivel en el punto de operación establecido por la base de reglas. Sin embargo, los cálculos que se requieren para obtener la salida deseada exigen un alto porcentaje de recursos para cómputo, lo que en ocasiones hace que los algoritmos no sean sencillos.

La anterior dificultad hace más complejo el proceso de implementación de un modelo difuso para controlar el nivel en el tanque empleando herramientas como Vensim, Ithink, entre otras. Andrade et al. (2011) proponen un software que permite combinar reglas de la teoría de conjuntos difusos mediante la dinámica de sistemas. La integración de dinámica de sistemas y sistemas de inferencia difusa aplicados en la evaluación de tecnologías la abordan Herrera (2014), Chen (2011) y Xu \& Li (2011). Por ende, la integración de metodologías de dinámica de sistemas y lógica difusa ha sido recientemente empleada para mejorar la toma de decisiones en la gestión empresarial. Esta alta exigencia en recurso para el procesamiento de los datos, presente en los controladores difusos, hace necesario encontrar herramientas que permitan simplificar los procesos de implicación, agregación y desfusificación presentes en los controladores difusos. Helbert (2009) propone una metodología llamada CBR (concresor basado en relaciones booleanas) para la implementación de sistemas de lógica difusa en los que se tienen en cuenta, los sensores, actuadores y las relaciones booleanas empleadas en el diseño de automatismos.

El esquema general para implementación del CBR está dado por cinco etapas. A continuación, se muestra la implementación de un controlador basado en CBR para el modelo del tanque, empleando reglas de operación, entradas y salidas definidas como sensores de nivel y válvulas para diferentes caudales.

En primer lugar, se definen cuatro sensores para detectar el nivel en el tanque, tres válvulas que regularán el flujo de entrada y un drenaje. Los sensores se asumen con las características que se muestran en la figura 10 y el modelo desarrollado con dinámica de sistemas en la figura 11.
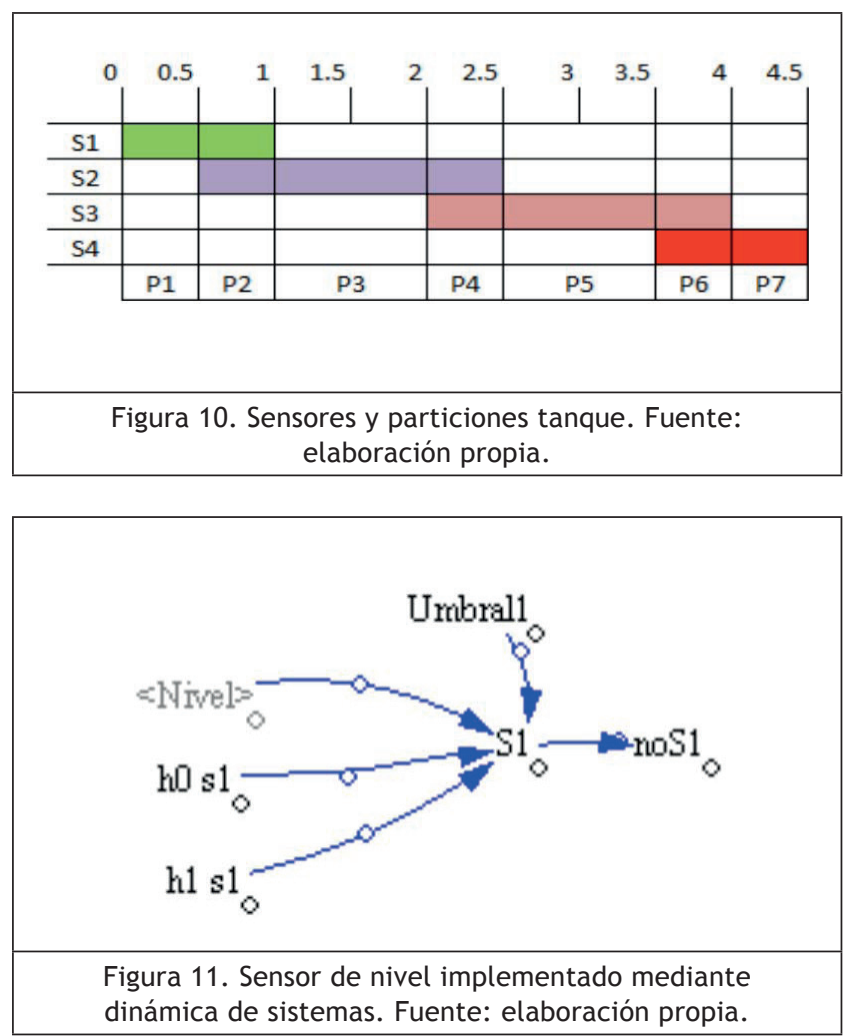
A partir de la región de activación de los cuatro sensores se pueden determinar las 7 zonas o particiones que se exponen en la tabla 3.

\begin{tabular}{|c|l|}
\hline \multicolumn{2}{|c|}{ Tabla 3. Particiones sensores. } \\
\hline PARTICIÓN & SENSORES ACTIVOS \\
\hline 1 & S1 \\
\hline 2 & S1 y S2 \\
\hline 3 & S2 \\
\hline 4 & S2 y S3 \\
\hline 5 & S3 \\
\hline 6 & S3 y S4 \\
\hline 7 & S4 \\
\hline \multicolumn{2}{|c|}{ Fuente: elaboración propia. } \\
\hline
\end{tabular}

La tabla 4 establece el estado (activación-desactivación) de las entradas y salidas para cada partición.

\begin{tabular}{|c|c|c|c|c|c|c|c|}
\hline \multicolumn{1}{|c|}{ Tabla 4. Tabla de valores de verdad. } \\
\hline & S1 & S2 & S3 & S4 & V1 & V2 & V3 \\
\hline P1 & 1 & 0 & 0 & 0 & 1 & 1 & 1 \\
\hline P2 & 1 & 1 & 0 & 0 & 0 & 0 & 1 \\
\hline P3 & 0 & 1 & 0 & 0 & 1 & 1 & 0 \\
\hline P4 & 0 & 1 & 1 & 0 & 0 & 1 & 0 \\
\hline P5 & 0 & 0 & 1 & 0 & 0 & 1 & 0 \\
\hline P6 & 0 & 0 & 1 & 1 & 1 & 0 & 0 \\
\hline P7 & 0 & 0 & 0 & 1 & 0 & 0 & 0 \\
\hline \multicolumn{17}{|c|}{ Fuente: elaboración propia. } \\
\hline
\end{tabular}

A continuación se determina el número de sensores y su forma de operación, y se establecen las reglas que van a caracterizar el funcionamiento deseado para la planta.

- Si el sensor S1 está activado, entonces V1V2 y V3 se activan.

- $\quad$ Si los sensores S1 y S2 se activan, entonces V1 y V2 se desactivan y V3 se activa.

- Si el sensorS2 se activa entonces, V1 y V2 se activan y V3 se desactiva.

- Si el sensor S2 se activa, entonces V1, V3 se activan y $\mathrm{V} 2$ se desactiva.

- Si los sensores S2 y S3 se activan, entonces V2 se activa y V1 y V3 se desactivan.

- Si el sensor S3 se activa, entonces V2 se activa y V1 y V3 se desactivan.
- Si los sensores S3 y S4 se activan, entonces V1 se activa y V2, V3 se desactivan.

- Si el sensor S4 se activa, entonces V1, V2 y V3 se desactivan.

Empleando herramientas para la determinación y simplificación de funciones booleanas, de acuerdo con la tabla 4, se encontraron las siguientes funciones que gobiernan la operación de las válvulas.

\begin{tabular}{|c|}
\hline 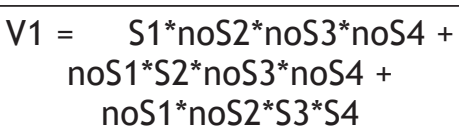 \\
\hline 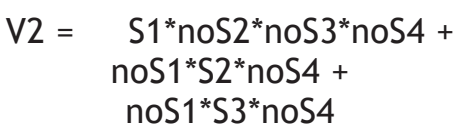 \\
\hline$V 3=S 1{ }^{*}$ nos $3 *$ nos 4 \\
\hline
\end{tabular}

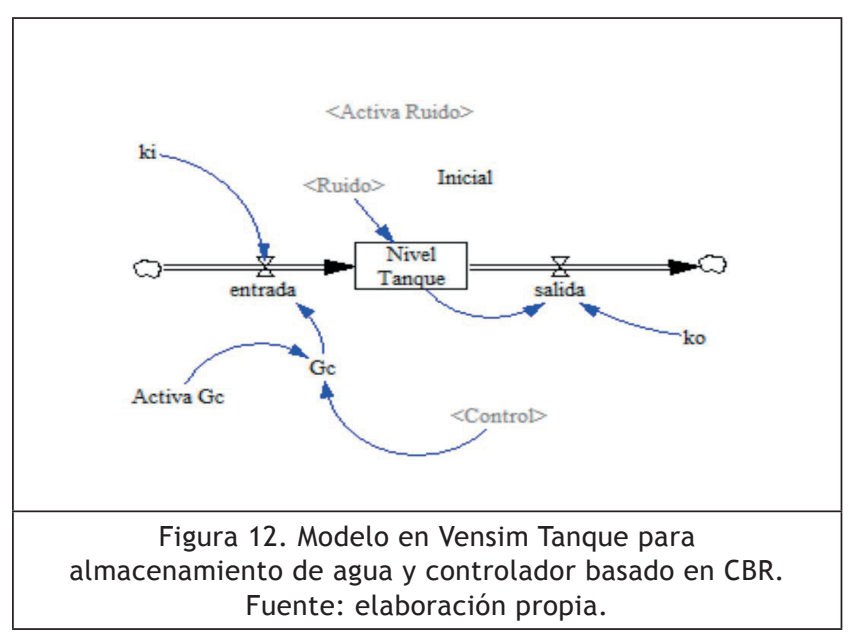

La implementación del modelo de control se muestra en las figura 12 y 13 , y el sistema de inferencia definido por las funciones V1, V2 y V3 se realizó empleando las operaciones de mínimo y máximo presentes en el modelo de dinámica de sistemas.

$$
\mathrm{V} 3=\operatorname{MIN}(\operatorname{MIN}(\mathrm{S} 1,1-\mathrm{S} 3), 1-\mathrm{S} 4))
$$

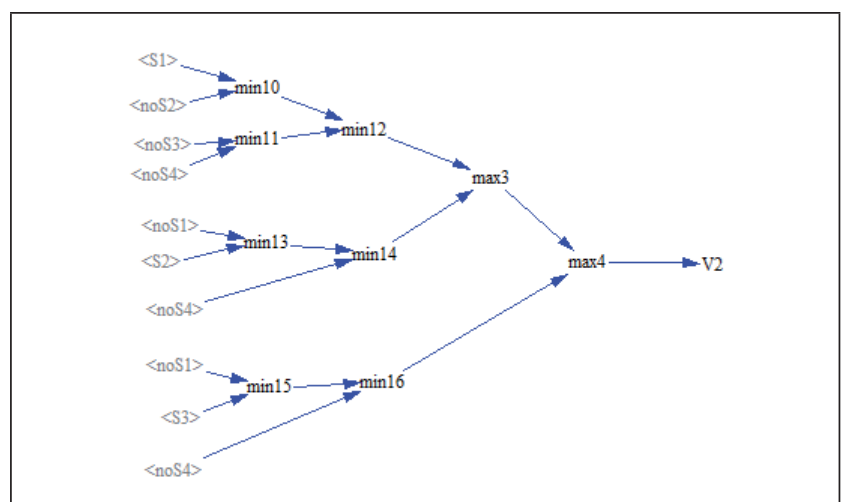

Figura 13. Implementación del sistema de inferencia según dinámica de sistemas. Fuente: elaboración propia. 
La acción de control está dada por la activación de las válvulas que regulan el flujo de entrada según las funciones determinadas y que dan origen al sistema de inferencia. En el modelo mostrado en la figura 14, las variables Kv1, Kv2 y Kv3 se usan para asignar una ganancia al flujo que entrega cada válvula. En este caso, se asumen valores iguales a 0,1, 0,3 y 0,6, respectivamente. Esto quiere decir que la válvula 3 entrega un flujo mayor al de las restantes válvulas.

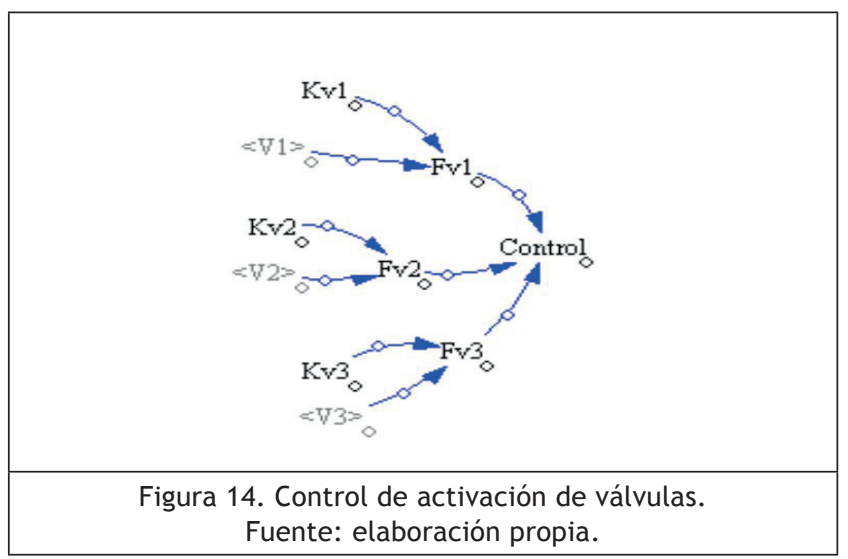

La respuesta del sistema se muestra en la figura 15, y al igual que con el controlador difuso implementado en Matlab, la altura en el tanque se mantiene en un valor próximo a $1 \mathrm{~m}$.
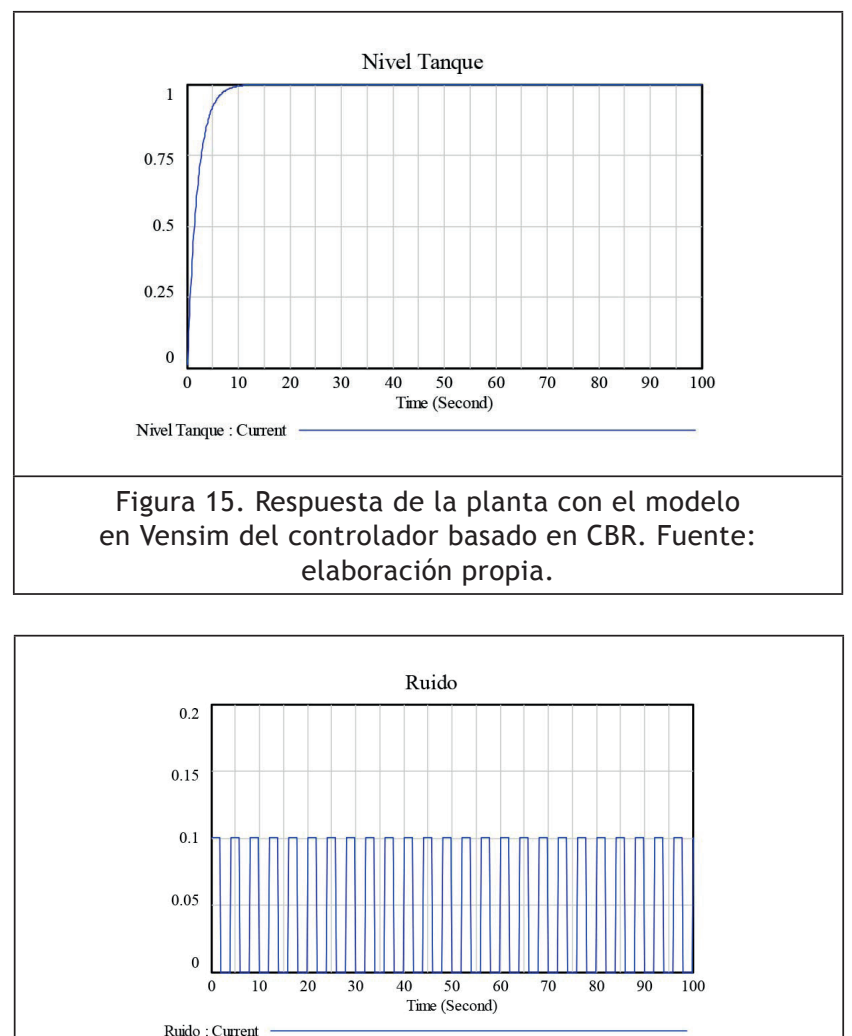

Figura 16. Señal perturbadora. Fuente: elaboración propia.
La inclusión de una señal perturbadora como la que aparece en las figuras 16 y 17 muestra una respuesta de la planta similar a la encontrada con el controlador difuso.

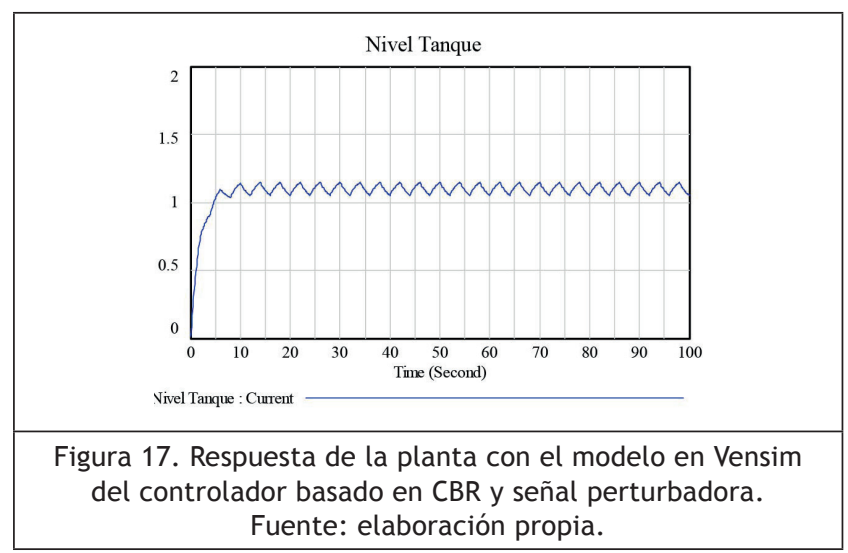

En resumen, los modelos desarrollados desde la dinámica de sistemas y la teoría de conjuntos difusos presentan resultados similares. Por lo tanto, el modelamiento de sistemas de control de un proceso físico se puede enseñar mejor por medio de la dinámica de sistemas, teniendo en cuenta la rigurosidad y formalismo de las técnicas empleadas en la ingeniería de control. El aporte de este artículo se enfoca en comparar las ténicas de control con Simulink y la metodología con dinámica de sistemas para mostrar las bondades del modelamiento de los procesos físicos mediante ésta y sus ventajas para comprender problemas dinámicos de una forma sencilla.

\subsection{Discusión de resultados}

Los resultados obtenidos con los modelos implementados mediante la dinámica de sistemas muestra una respuesta similar a los creados en Matlab, debido a que la formalidad matemática se resuelve a partir de las variables de flujo que presentan los modelos con dinámica de sistemas. Esto se debe a que al aplicar las herramientas del pensamiento sistémico (diagramas de ciclo causal, arquetipos y software), así como las interrelaciones, causas y efectos detectados para un problema, hecho, fenómeno o proceso, es posible crear una estructura en la cual sus componentes se alimentan de forma recíproca generando crecimiento, disminución o el movimiento natural hacia un estado de equilibrio.

El uso de técnicas de control y simulación permiten entender el comportamiento de los procesos físicos. Por lo tanto, en los procesos de aprendizaje, entender el comportamiento por medio de la simulación se convierte en una estrategia educativa y de investigación (Herrera, Molano \& Sandoval, 2014; Andrade et al., 2011a; Arismendi et al., 2011; Beall \& Ford, 2011). Richmond (1993) plantea una reflexión del pensamiento sistémico en la educación en Estados Unidos. A partir 
de este trabajo se generan propuestas como las de Davidsen et al. (1993), Saeed (1993) y Simons (1993), que utilizan la dinámica de sistemas como metodología en las actividades de educación y que contribuyen en los procesos y análisis del aprendizaje.

Los modelos clásicos desarrollados permiten apropiar conceptos de control de los procesos con técnicas de simulación. La comparación de los métodos empleados permite una mejor abstracción de los conceptos básicos que se desarrollan para el diseño de los sistemas de control automatizados. Los modelos presentados muestran el formalismo matemático requerido y los posibles cambios que se pueden proponer con el propósito de generar políticas de control alrededor de un proceso físico en varios escenarios.

\section{CONCLUSIONES}

En general, los conceptos de la dinámica de sistemas facilitan las tareas para representar, evidenciar y comprender sistemas dinámicos complejos por medio de modelos sencillos. El artículo presenta tres casos que fueron modelados clásicos y permiten un mejor nivel de extracción de conceptos cuando se emplea la metodología propuesta por la dinámica de sistemas.

Los modelos clásicos de procesos físicos permiten apropiar los conceptos de control y simulación de los puntos de operación. La comparación permite entender el modelamiento de los sistemas y las estrategias alrededor de un mejor modelado que integra los sistemas dinámicos y la dinámica de sistemas. La relación entre éstos se puede apreciar en la comparación realizada con los modelos propuestos. Las diferencias formales (matemáticas) se abordan para revisar la eficiencia computacional y estrategia de modelamiento.

Por otra parte, la integración de diversos métodos es una tendencia en varios trabajos desarrollados con enfoques de dinámica de sistemas. Por consiguiente, hasta el momento las aplicaciones en dicha integración han sido particulares a diferentes fenómenos o representaciones reales.

\section{REFERENCIAS}

1. Andrade, H., Lince, E., Hernández, A. \& Monsalve, A. (2011). Evolución: herramienta software para modelado y simulación condinámica de sistemas. Revista de Dinámica de Sistemas, 5(1).

2. Andrade, H., Luque, G. \& Lince, E. (2010). Sistemas de inferencia difusa en el modelado con conocimiento aproximado. Proceeding
Conferences, 8.o Congreso Latinoamericano de Dinámica de Sistemas.

3. Andrade, H., Maestre, G. \& López, G. (2011a). Desarrollo de competencias en la toma de decisiones con dinámica de sistemas una experiencia en el aula. Proceeding Conferences, 9.0 Congreso Latinoamericano de Dinámica de Sistemas.

4. Arismendi, J. \& Andrade, H. (2011). Reflexiones sobre el diseño de experimentos con dinámica de sistemas en educación. Proceeding Conferences, 9.0 Congreso Latinoamericano de Dinámica de Sistemas.

5. Aracil, J. (1997). Dinámica de sistemas. Madrid, España: Alianza Editorial.

6. Beall, A. \& Ford, A. (2011). Learning from system dynamics simulations: time compression in system dynamic versus time dilation in power system simulators. Proceeding Conference. The 29th International Conference of the System Dynamics Society.

7. Caselles, A. (2008). Modelización y simulación de sistemas complejos. Universidad de Valencia.

8. Charles, M. C. (2001). Modeling and analysis of dynamic systems (3.a ed.). Estados Unidos: Editorial John Willey \& Songs.

9. Chen, Y. (2011). Understanding technology adoption through system dynamics approach: a case study of RFID technology. Proceedings - 2011 IFIP 9th International Conference on Embedded and Ubiquitous Computing, EUC 2011, art. no. 6104551, pp. 366-371.

10. Davidsen, P., Bjurklo, M. \& Wikstrom, H. (1993). Introducing system dynamics in schools: the Nordic experience. System Dynamic Review.

11. Del Brío, M. (2001). Redes neuronales y sistemas difusos (2.a ed.). México, D.F.: Alfaomega.

12. Espitia, C. \& Helbert, E. (2009). Aplicación del concresor basado en relaciones booleanas para sistemas de lógica difusa tipo 2. Tesis de maestría, Universidad Distrital Francisco José de Caldas, Bogotá.

13. Forrester, J. (1994). System dynamics systems thinking soft OR. System Dynamics Review.

14. Hans, F. U. (2006). System dynamics modeling in science and engineering. Department of Physics and Mathematics, Zurich University of Applied Sciences at Winterthur. 
15. Herrera, R. \& Echavarría, C. (2008). Forraje hidropónico como alternativa de innovación y tecnología en los procesos de producción agroindustrial sustentable. Proceeding Conference Ibergecyt 2008. La Habana, Cuba.

16. Herrera, R. \& Bonilla, R. (2009). Simulation strategies consider in the agro-industry production. Proceeding Conference Seventh Latin American and Caribbean Conference for Engineering and Technology.

17. Herrera R.\& Orjuela Castro, J. A. (2014). Perspectiva de trazabilidad en la cadena de suministros de frutas: un enfoque desde la dinámica de sistemas. Ingeniería (0121-750X), 19(2).

18. Herrera M. (2014). Modelo para la implementación de tecnología de trazabilidad RFID en la cadena de suministro frutícola en las operaciones de picking con un enfoque integral y dinámico difuso. Tesis de maestría en Ingeniería Industrial. Bogotá, D.C.: Universidad Distrital Francisco José de Caldas.

19. Herrera, M., Molano, J. \& Sandoval, H. (2014). Diseño de estrategias y políticas e investigación en la educación bajo dinámica de sistemas. Revista Inventum, 17, pp. 23-31.

20. Isee Systems (2012). Temperature control, A Learning Laboratory created with Stella. Recuperado de http://forio.com/simulate/ simulation/netsim/temperature-controller/.

21. Mohapatra, P. \& Sharma, S. (1985). Synthetic design of policy decisions in system dynamic models: a modal control theoretical approach. System Dynamic Review.

22. Morecroft, J. \& Sterman, J. (1994). Modeling for learning. Portland, OR: Productivity Press.

23. Norgaard, O. M. (2000). Neural networks for modelling and control of dinamic systems. London: Springer-Verlag.
24. Ogata, K. (1998). Ingeniería de control moderna. México: Editorial Pearson.

25. Richmond, B. (1993). Systems thinking: critical thinking skills for the 1990 and beyond. System Dynamics Review.

26. Sabounchi, N., Triantis, K., Sarangi, S. \& Liu, S. (2011). Fuzzy modeling of linguistic variables in a system dynamics context. Proceeding Conferences The 29th International Conference of Dynamic Society.

27. Saeed, K. (1993). Bringing experimental learning to social science: simulation laboratory on issues of economic development. System Dynamic Review.

28. Shannon, C. E. (1938). A symbolic analysis of relay and switching circuits. American Institute of Electrical Engineers, 57(12), pp. 713-723, Versión de junio de 2009, New York.

29. Sterman, J. D. (2000). Business dynamics: systems thinking and modeling for a complex world. McGraw Hill.

30. Tessem, B. \& Davidsen, P. (1994). Fuzzy system dynamics: an approach to vague and qualitative variables in simulation. System Dynamics Review.

31. Vensim User Guide V5 (2007). Ventana Simulation Environment DSS, Professional, PLE Plus, PLE. Recuperado de http://www.vensim.com/ documentation.html.

32. Wang, Li X. (1997). A course in fuzzy systems to control. New York: Prentice Hall International.

33. Wolstenholme, E. (1986). Algorithmic control optimal control problems with system dynamics. System Dynamic Review.

34. Xu, J. \& Li, X. (2011). Using system dynamics for simulation and optimization of one coal industry system under fuzzy environment. Expert Systems with Applications, 38 (9), pp. 11552-11559. 